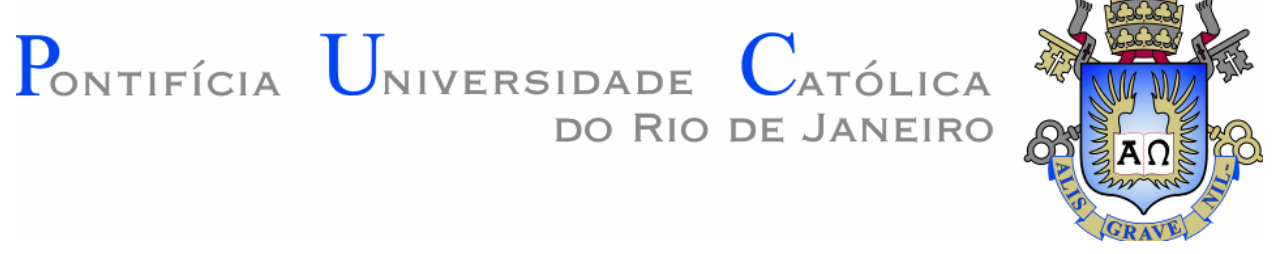

Gerson Ortiz Gallo

\title{
Avaliação da eficácia da alumina como revestimento do aço P92 na corrosão em altas temperaturas
}

\section{Dissertação de Mestrado}

Dissertação apresentada como requisito parcial para obtenção do grau de Mestre pelo Programa de Pós-graduação em Engenharia de Materiais e de Processos Químicos e Metalúrgicos do Departamento de Engenharia Química e de Materiais do Centro Técnico Cientifico da PUC-Rio.

Orientador: Prof. Fernando Cosme Rizzo Assunção Coorientador: Dr Maurício De Jesus Monteiro

Rio de Janeiro

Abril 2018 


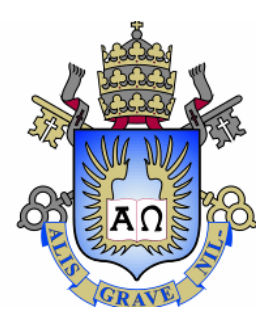

Gerson Ortiz Gallo

\section{Avaliação da eficácia da alumina como revestimento do aço P92 na corrosão em altas temperaturas}

Dissertação apresentada como requisito parcial para obtenção do grau de Mestre pelo Programa de Pós-graduação em Engenharia de Materiais e de Processos Químicos e Metalúrgicos do Departamento de Engenharia de Materiais do Centro Técnico Cientifico da PUC-Rio. Aprovada pela comissão examinadora abaixo assinada

\section{Prof. Fernando Cosme Rizzo Assunção Orientador Departamento de Engenharia de Materiais - PUC-Rio \\ Dr. Maurício de Jesus Monteiro Coorientador} Instituto Nacional de Tecnologia, Ministério de Ciência, Tecnologia e Inovação (INT)

Profa. Ivani de Souza Bott Departamento de Engenharia de Materiais - PUC-Rio

Dr. leda Maria Viera Caminha Instituto Nacional de Tecnologia, Ministério de Ciência, Tecnologia e Inovação (INT)

Prof. Marcio da Silveira Carvalho Coordenador Setorial Centro Cientifico- PUC-Rio (INT) 
Todos os direitos reservados. É proibida a reprodução total ou parcial do trabalho sem autorização da universidade, do autor e do orientador.

\section{Gerson Ortiz Gallo}

Formado em engenharia de petróleo pela universidade industrial de Santander (UIS) (Bucaramanga- Colômbia) em 2014 na área de gestão ambiental em exploração e produção de hidrocarbonetos (UPSTREAM). Especializando-se atualmente nas áreas de: Ciência dos materiais, corrosão em altas temperaturas e caracterização de materiais.

Ficha Catalográfica

Ortiz Gallo, Gerson

Avaliação da eficácia da alumina como revestimento do aço P92 na corrosão em alta temperaturas / Gerson Ortiz Gallo ; orientador: Fernando Cosme Rizzo Assunção ; co-orientador: Maurício de Jesus Monteiro. 2018.

86 f. : il. color. ; $30 \mathrm{~cm}$

Dissertação (mestrado)-Pontifícia Universidade Católica do Rio de Janeiro, Departamento de Engenharia Química e de Materiais, 2018.

Inclui bibliografia

1. Engenharia de Materiais - Teses. 2. Engenharia Química - Teses. 3. Corrosão em alta temperaturas. 4. Ligas Fe-Cr. 5. Oxicombustão. 6. Recobrimento. 7. Alumina. I. Assunção, Fernando Cosme Rizzo. II. Monteiro, Maurício de Jesus. III. Pontifícia Universidade Católica do Rio de Janeiro. Departamento de Engenharia Química e de Materiais. IV. Título. 


\section{Agradecimentos}

Primeiramente agradeço enormemente a minha família em especial a meus pais que me ajudaram e apoiaram em todo este processo.

Ao professor Fernando Rizzo pela orientação, conselhos e apoio durante o desenvolvimento do trabalho.

Ao Dr. Mauricio Monteiro, pela gentileza em me ajudar e disposição no projeto.

Para o Professor Alex Kranzmann e a equipe da BAM pelo projeto

Agradeço a Leydi por seu compromisso, dedicação, e apoio moral incondicional em todo o caminho em minha pesquisa.

Meus amigos Loren, Raul que me ajudaram ainda nos momentos mais difíceis.

Ao Centro de Caracterização em Nanotecnologia para Materiais e Catálise (CENANO) do Instituto Nacional de Tecnologia (INT) especialmente para Rachel, Francisco e David, que me ajudaram e colaboraram na minha aprendizagem.

Ao senhor Robson do INT por compartilhar seu conhecimento e experiência que contribuiu na minha formação.

À PUC-Rio pela oportunidade do mestrado.

À CNPq pelo contínuo apoio financeiro, o que tornou possível esse trabalho. 


\section{Resumo}

Ortiz Gallo, Gerson; Assunção, Fernando Cosme Rizzo (Orientador); Monteiro, Maurício De Jesus (Coorientador). Avaliação da eficácia da alumina como revestimento do aço $P 92$ na corrosão em altas temperaturas. Rio de Janeiro, 2018. 86p. Dissertação de Mestrado Departamento de Engenharia Química e de Materiais, Pontifícia Universidade Católica do Rio de Janeiro.

A redução na emissão de $\mathrm{CO}_{2}$ na atmosfera é um dos desafios atuais mais importantes enfrentados por diferentes indústrias no mundo. A geração de energia através da queima de combustíveis fósseis utilizando oxigênio puro [1], também chamada de tecnologia de oxicombustão, é uma opção utilizada pelas indústrias de geração de energia para minimizar os índices de concentração de $\mathrm{CO}_{2}$ até emissão ZERO [1,2]Inevitavelmente, as tubulações dos trocadores de calor que conformam aquelas plantas de energia, sofrem corrosão em contato com os gases de combustão $\left(\mathrm{H}_{2} \mathrm{O}-\mathrm{CO}_{2}\right)$ em altas temperaturas. Estas tubulações, fabricadas com aço ou com ligas ferro-cromo vem sendo utilizadas no intuito de reduzir a corrosão. Tubulações com revestimentos/recobrimentos protetores tem sido também considerados para aumentar a resistência a processos corrosivos sem requerer o emprego de ligas de alto custo. Um revestimento adequado deve ser inerte e estável em altas temperaturas como é o caso dos materiais cerâmicos. A alumina, sendo um material estável em ambientes característicos do processo de oxicombustão apresenta características favoráveis para esta proteção [3]. Amostras de $\mathrm{Fe}-\mathrm{Cr}$ foram oxidadas sob condições que simularam processos de Oxicombustão nos laboratórios do Bundesanstalt Für Materialforschung Und-Prüfung (BAM) Berlim-Alemanha. O processo ocorreu a $650{ }^{\circ} \mathrm{C}$ durante $2000 \mathrm{~h}$, em atmosfera contendo $60 \% \mathrm{CO}_{2}$, $30 \% \mathrm{H}_{2} \mathrm{O}, 7 \% \mathrm{~N}_{2}, 2 \% \mathrm{O}_{2}$ e $1 \% \mathrm{SO}_{2}$. A caracterização microestrutural das amostras oxidadas foi realizada nos laboratórios do instituto Nacional de Tecnologia (INT), utilizando técnicas de Microscopia Eletrônica de Varredura (MEV) e Espectroscopia por Dispersão de Energia de Raios- X (EDS). Foram produzidos riscos na superfície do revestimento de $\mathrm{Al}_{2} \mathrm{O}_{3}$, previamente aos testes de oxidação, para simular falhas no revestimento; além da eficácia do revestimento de alumina, foi também avaliado o papel do cromo como meio protetor contra a corrosão e de outros elementos de liga no processo de oxidação do aço P92, buscando identificar 
o mecanismo de oxidação. Como resultado do fluxo dos gases de oxicombustão há formação de camadas de $\mathrm{Fe}_{3} \mathrm{O}_{4}, \mathrm{Fe}_{2} \mathrm{O}_{3}$ e cromo-espinélio nas ligas sem revestimento. As amostras que possuíam o revestimento de alumina apresentaram ótima proteção, com resultados de uma alta eficácia, enquanto as amostras que tinham riscos apresentaram uma oxidação interna de oxidação cromo-espinélio protetor na matriz logo abaixo da região afetada.

\section{Palavras-chave}

Corrosão em Alta Temperaturas; Ligas Fe-Cr; Oxicombustão; Recobrimento; Alumina. 


\section{Abstract}

Ortiz Gallo, Gerson; Assunção, Fernando Cosme Rizzo (Orientador); Monteiro, Maurício De Jesus (Coorientador). Evaluation of the efficiency of alumina-coated P92 steel to high temperatures corrosion. Rio de Janeiro, 2018. 86p. Dissertação de Mestrado - Departamento de Engenharia Química e de Materiais, Pontifícia Universidade Católica do Rio de Janeiro.

The reduction in the emission of $\mathrm{CO}_{2}$ in the atmosphere is one of the most important problems faced by different industries in the world. The generation of energy from oxyfuel oxygen, [1], also called Oxyfuel technology, is a matter of $\mathrm{CO}_{2}$ emission for ZERO [1,2]. Inevitably, as pipes of the heat exchangers that make up these power plants, they suffer corrosion in contact with the flue gases $\left(\mathrm{H}_{2} \mathrm{O}-\mathrm{CO}_{2}\right)$ at high temperatures. These pipes, made of steel or iron-chromium alloys, are not used instead of reducing corrosion. Pipes with protective coatings have already been submitted to increase resistance to corrosive processes without requiring the use of high cost alloys. One which must be inert and static at high temperatures is the case of ceramic materials. An alumina, being a suitable material to the environments characteristic of the Oxyfuel process, presents characteristics favorable to the protection [3]. Fe-9Cr samples were oxidized under conditions that simulated Oxyfuel processes in the laboratories of the Bundesanstalt Für Materialforschung Und-Prüfung (BAM) Berlin-Germany. The process occurred at $650{ }^{\circ} \mathrm{C}$ for $2000 \mathrm{~h}$, in an atmosphere containing $60 \% \mathrm{CO}_{2}, 30 \% \mathrm{H}_{2} \mathrm{O}, 7 \% \mathrm{~N}_{2}, 2 \%$ $\mathrm{O}_{2}$ and $1 \% \mathrm{SO}_{2}$. The microstructural characterization of the oxidized samples was performed in the laboratories of the National Institute of Technology (INT), using Scanning Electron Microscopy (SEM) and X-ray Energy Dispersion Spectroscopy (EDS) techniques. Risks were produced on the $\mathrm{Al}_{2} \mathrm{O}_{3}$ coating surface, prior to the oxidation tests, to simulate coating failures; besides the effectiveness of the alumina coating, the role of chromium as a protective medium against corrosion and other alloying elements in the oxidation process of P92 steel was also evaluated, in order to identify the oxidation mechanism. As a result of the flow of oxyfuel gases, $\mathrm{Fe}_{3} \mathrm{O}_{4}$, $\mathrm{Fe}_{2} \mathrm{O}_{3}$ and chromium-spinel layers are formed in the alloys without coating. The samples that had the alumina coating presented optimum protection, with results of 
a high efficiency, while the samples that had scratches presented an internal oxidation of protective chromium-spinel oxidation in the matrix just below the affected region.

\section{Keywords}

High Temperature Corrosion; Fe-Cr Alloys; Oxyfuel; Coating; Alumina. 


\section{Sumário}

1 Introdução

2 Revisão Bibliográfica $\quad 18$

2.1. Sequestro de $\mathrm{CO}_{2} \quad 18$

2.1.1. Captura pós-combustão 19

2.1.2. Captura pré-combustão 19

2.1.3. Captura Oxicombustão 20

2.2. Processo de oxidação em altas temperaturas 22

2.3. Cinética de oxidação 23

2.3.1. Cinética de oxidação linear. 23

2.3.2. Cinética de oxidação parabólica. 24

2.3.3. Cinética de oxidação logarítmica. 25

2.4. Revestimentos 25

2.4.1. Revestimento Sol Gel Alumina 27

2.5. Ligas $\mathrm{Fe}-9 \% \mathrm{Cr} 28$

2.6. Carburização. 31

2.7. Sulfetação 31

2.8. Processo de oxidação em metais 32

2.8.1. Oxidação do Ferro. 33

2.8.1. Oxidação na liga Ferro-Cromo. 36

2.8.2. Atmosfera contendo vapor de $\mathrm{H}_{2} \mathrm{O} 36$

2.8.3. Atmosfera contendo CO2 39

2.8.4. Atmosfera de Oxicombustão 41

3 Materiais e Métodos $\quad 44$

3.1. Materiais e gases 46

3.1.1. Gás de Oxicombustão 46

3.2. Metodologia 46

3.2.1. Preparação do Sol Gel 48

3.2.2. Metalografia 49

3.2.3. Preparação das amostras no microscópio eletrônico de varredura MEV. 
4 Análise e Discussão Dos Resultados 54

4.1. Amostra P2 56

4.1.1. Análise superficial da amostra P2 56

4.1.2. Observação da seção transversal da amostra P2 60

4.2. Amostra P3 66

4.2.1. Análise da superfície da amostra P3 66

4.2.2. Observação da seção transversal da amostra P3 68

4.3. Amostra P4 71

4.3.1. Análise da superfície amostra P4 71

4.3.2. Observação da seção transversal da amostra P4 72

$\begin{array}{ll}5 \text { Conclusões } & 78\end{array}$

6 Referências Bibliográficas $\quad 80$ 


\section{Lista de figuras}

Figura 1: Concentração atmosférica de $\mathrm{CO} 2$ nos últimos anos[6].......... 18

Figura 2: Esquema dos processos pós-combustão, pré-combustão

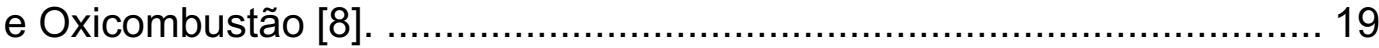

Figura 3: Esquema do processo pré-combustão. ................................. 20

Figura 4: Esquema do processo de Oxicombustão de uma planta de geração de energia [13] . ............................................................... 21

Figura 5: Seção transversal de uma camada de óxido formada na superfície de um metal ou liga [21]. ............................................. 22

Figura 6: Cinética de oxidação linear, parabólico e logarítmico [22] ........ 23

Figura 7: Imagem ilustrativa liga com revestimento. ............................. 26

Figura 8: Processo de dispersão de partículas pelo método de sol-gel... 28

Figura 9: Variação em peso/tempo do óxido formado no aço P92

em diferentes temperaturas oxidado com gás oxicombustão.

Figura 10: MEV Seção transversal de amostra do aço P92 oxidadas em $\mathrm{CO}_{2} / \mathrm{H}_{2} \mathrm{O}$ para diferentes tempos de exposição a $550^{\circ} \mathrm{C}$. Tempo de exposição de 24h, 100h, 250h, 200h, 750h, 1000h [8]. 30

Figura 11 MEV Seção transversal de amostra do aço P92 oxidadas em $\mathrm{CO}_{2} / \mathrm{H}_{2} \mathrm{O}$ a $600{ }^{\circ} \mathrm{C}, 650^{\circ} \mathrm{C}$ e $700{ }^{\circ} \mathrm{C}$. Tempo de exposição 1000h [8].

Figura 12: Sulfetos em aço P92 exposto a gases de oxicombustão em $580^{\circ} \mathrm{C}$ e $650^{\circ} \mathrm{C}$ [37]......

Figura 13: Esquema do Processo de oxidação em altas

temperaturas quanto uma superfície metálica entra em contato com o oxigênio [8] 33

Figura 14: Diagrama de fases ferro-oxigênio [21]. 34

Figura 15: Mecanismo de oxidação do Ferro com a formação das camadas de $\mathrm{FeO}$ (Wustita), Fe3O4(Magnetita), e Fe2O3(Hematita) respectivamente [13]. 35

Figura 16: Efeito do cromo na resistência à oxidação e morfologia do óxido de ligas $\mathrm{Fe}, \mathrm{Fe}-2 \% \mathrm{Cr}$, $\mathrm{Fe}-9 \% \mathrm{Cr}$ respectivamente. 36

Figura 17: Sequência de formação de camadas na oxidação com 
Figura 18: Sequência de formação de camadas na oxidação com presença do vapor d'água[41] 38

Figura 19: Formação de vazios em uma liga $\mathrm{Fe}-\mathrm{Cr}$ produzidos durante a oxidação com vapor de água

Figura 20: Formação de carbonetos em uma liga P92 oxidada a $610^{\circ} \mathrm{C}$ a $1000 \mathrm{~h}[36]$ 40

Figura 21: Mecanismo de oxidação em uma atmosfera de $\mathrm{CO}_{2}[13]$ 41

Figura 22: Formação de camadas de óxidos após oxidação com gás de oxicombustão. Atmosfera de $650^{\circ} \mathrm{C}$ por $300 \mathrm{~h}$ em uma liga $\mathrm{Fe} 9-12 \% \mathrm{Cr}$ [35].

Figura 23: Relação ganho de peso Vs Temperatura da oxidação com Ar-50\% $\mathrm{CO} 2$ a 250h [48].

Figura 24: Resumo das condições de oxidação e imagens das amostras após os ensaios de oxidação 45

Figura 25: Composição em \%Vol do gás Oxicombustão [35]. 46

Figura 26: Fornos tubulares utilizados na oxidação das amostras P2, P3, P4 [8].

Figura 27: Desenho Esquemático dos ensaios de oxidação com o gás oxicombustão durante $2000 \mathrm{~h}$ a $650^{\circ} \mathrm{C}$. O experimento foi realizado numa pressão de 80 Bar.

Figura 28: Representação do processo da preparação da técnica Sol-Gel por imersão para a adição do revestimento de Alumina 1) dispersão de boehmita em etanol. 2) adição de aglutinante PVB em sol. 3) dissolução ao banho-maria. 4) imersão da amostra no sol. 5) secar amostra a temperatura ambiente. 6) primeirosegundo aquecimento num forno $120^{\circ} \mathrm{C}$ por $5 \mathrm{~min}$ e $650^{\circ} \mathrm{C}$. Por 30 min. 49

Figura 29: Imagens dos materiais e equipamento utilizados no embutimento a frio das amostras. 50

Figura 30: Imagens dos materiais e equipamento utilizados no embutimento a quente das amostras. 51

Figura 31: Colocação da amostras no microscópio eletrônico de varredura, os números correspondem às amostras da seguinte maneira: 
1) $P 2$ 2 2) $P 3$, 3) $P 4$.

52

Figura 32: Processo de metalização das amostras com prata e fluxo de gás de argônio por 2,5 min

Figura 33: Micrografias obtidas por estereoscopia da superfície amostras $\mathrm{P}$ 1) aço material base $\mathrm{P} 2$ ) aço base oxidado, P3) aço base com revestimento de alumina e oxidado e $\mathrm{P} 4$ ) aço base com um risco no revestimento de alumina antes da oxidação. 55

Figura 34: Superfície do aço P92 oxidado com gás oxicombustão a $650^{\circ} \mathrm{C}$ por $2000 \mathrm{~h}$. Imagem de MEV com uma magnificação de 200x

Figura 35: Micrografia obtida por MEV operando a $15 \mathrm{kV}$, utilizando detector de elétrons secundários e magnificação de $10000 \mathrm{x}$.

Figura 36: Micrografia obtida por MEV operando a $15 \mathrm{kV}$, utilizando detector de elétrons secundários e magnificação de $10000 \mathrm{x}$. 58

Figura 37: Micrografia obtida por MEV operando a $15 \mathrm{kV}$, utilizando detector de elétrons secundários e magnificação de $20000 \mathrm{x}$. 59

Figura 38: MEV Seção transversal de amostra do aço P92 oxidada a $650^{\circ} \mathrm{C}$ por $2000 \mathrm{~h}$. Camada interna: óxidos contendo Ferro e Cromo; Camada externa: óxidos de Ferro.

Figura 39: Caracterização metalográfica da seção transversal das camadas de óxido formadas em aço $\mathrm{P} 92$ a $650^{\circ} \mathrm{C}$ por $2000 \mathrm{~h}$. 62

Figura 40: Formação de camadas de óxido hematita, magnetita e cromo-espinélio num aço $\mathrm{P} 92$ oxidado a $650^{\circ} \mathrm{C}$ por $2000 \mathrm{~h}$.

Figura 41: Seção transversal ilustrando a formação das camadas de óxido no aço $\mathrm{P} 92$ oxidada a $650^{\circ} \mathrm{C}$ por $2000 \mathrm{~h}$ numa atmosfera de oxicombustão. Camada interna (Cromo espinélio $\mathrm{FeCr}_{2} \mathrm{O}_{4}$ ), camada externa $\left(\mathrm{Fe}_{3} \mathrm{O}_{4}\right.$ e $\left.\mathrm{Fe}_{2} \mathrm{O}_{3}\right)$. 64

Figura 42: Mapeamento MEV/EDS da seção transversal do aço P92 oxidado a $650^{\circ} \mathrm{C}$ por $2000 \mathrm{~h}$ numa atmosfera de oxicombustão. 65

Figura 43: Óxido formado no canto da amostra P92 apos oxidação a $650^{\circ} \mathrm{C}$ a $2000 \mathrm{~h}$

Figura 44: Micrografia da amostra P3 obtida por MEV operando a $15 \mathrm{kV}$, utilizando detector de elétrons secundários e magnificação de $200 \mathrm{x}$. 
Figura 45: Aspecto superficial da amostra P3 obtida por MEV operando a $15 \mathrm{kV}$, utilizando detector de elétrons secundários e magnificação de $2000 x$

Figura 46: (MEV) seção transversal do aço P92 com revestimento de $\mathrm{Al}_{2} \mathrm{O}_{3} \mathrm{e}$ oxidada a $650^{\circ} \mathrm{C}$ por $2000 \mathrm{~h}$. Operando a $20 \mathrm{kV}$, utilizando detector de elétrons secundários e magnificação de $3000 x$ 68

Figura 47: Mapeamento MEV/EDS da seção transversal do aço P92 com revestimento de alumina e oxidado com gás oxicombustão $20 \mathrm{Kv}$ e magnificação de 5000x . 69

Figura 48: Mapeamento MEV/EDS da seção transversal do aço P92 com revestimento de alumina e oxidado com gás oxicombustão $20 \mathrm{Kv}$ e magnificação de 2000x.

Figura 49: MEV da superfície do aço P92 com revestimento de alumina e riscado antes da oxidação a $650^{\circ} \mathrm{C}$ por $2000 \mathrm{~h}$, numa atmosfera de oxicombustão.

Figura 50: MEV seção transversal do aço P92 com revestimento de alumina e riscado antes da oxidação a $650^{\circ} \mathrm{C}$ por $2000 \mathrm{~h}$

Figura 51: MEV seção transversal do aço $\mathrm{P92}$ oxidada a $650^{\circ} \mathrm{C}$ por 2000h. Óxido interno formado produto da oxidação.

Figura 52: MEV seção transversal do aço $\mathrm{P92}$ oxidada a $650^{\circ} \mathrm{C}$ por 2000h. Camada interna rica em cromo, camada externa rica em ferro... 74 Figura 53: Mapeamento de EDS da seção transversal do aço P92 com revestimento de alumina risco 1 e oxidado a $650^{\circ} \mathrm{C}$ por $2000 \mathrm{~h}$ numa atmosfera de oxicombustão 75

Figura 54: MEV seção transversal do aço $\mathrm{P92}$ oxidada a $650^{\circ} \mathrm{C}$ por 2000h. Camada interna rica em cromo, camada externa rica em ferro. .. 76 Figura 55: Mapeamento de EDS da seção transversal do aço P92 com revestimento de alumina risco 2 e oxidado a $650^{\circ} \mathrm{C}$ por $2000 \mathrm{~h}$ numa atmosfera de oxicombustão 


\section{Lista de Tabelas}

Tabela 1: Composição da liga P92 Ferrítico-Martensítico.[25] ................ 46

Tabela 2: Composição química ( wt.\%) obtida por EDS da superfície da amostra P2 59

Tabela 3: Composição química ( wt.\%) obtida por EDS da superfície da amostra P2 com magnificação de 2000x 60

Tabela 4: Composição química ( wt. \%) obtida por EDS na seção transversal da amostra P2 apresentada na Figura 44. 64

Tabela 5: Composição química ( wt.\%) obtida por EDS da superfície da amostra P3 com magnificação de 2000x 68

Tabela 6: Composição química (wt.\%) obtida por EDS da superfície da amostra P4.

Tabela 7: Composição química ( wt.\%) obtida por EDS do óxido formado no risco 1

Tabela 8: Composição química ( wt.\%) obtida por EDS do óxido formado no risco 2 